\title{
Comparison of Biometric Measurements Using Dielectric Spectroscopy and Blood Flow Meter
}

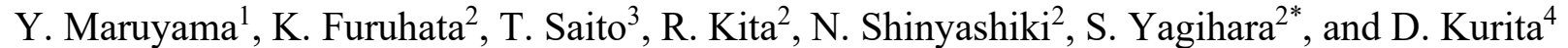 \\ 1 Graduate School of Science and Technology, Tokai University, Hiratsuka, Kanagawa 259-1292, Japan \\ 2 School of Science, Tokai University \\ 3 Graduate School of Science, Tokai University \\ 4 IT Education Center, Tokai University \\ *Fax: 81-463-50-2013, e-mail: yagihara@keyaki.cc.u-tokai.ac.jp
}

\begin{abstract}
The blood flow of the living body observed by ultrasonic blood flow meter and laser blood flow meter were analyzed whether these results are also reflected in relaxation phenomena obtained from dielectric measurements by impedance analyzer. The interface polarization of red blood cells (RBCs) obtained by dielectric measurements in vivo clearly indicated that the RBC number density increased by sprint. This explanation was confirmed from comparison with the results obtained by ultrasonic blood flow meter and laser blood flow meter. As the blood vessels dilated due to sprint, the amount of blood pumped out increased and RBCs observed by dielectric measurements increased. As the blood volume increased, the mean blood flow velocity was found to be lower than that at rest. The results suggest the blood state in the living body can be obtained by dielectric spectroscopy.

Key words: dielectric spectroscopy, in vivo, non-invasive, RBCs, blood flow
\end{abstract}

\section{INTRODUCTION}

Inside the living tissue, water structures taking complex high-order structures through hydrogen bonds with various biological molecules are constantly ever-changing in the picosecond time domain. These have been observed in the $\mathrm{GHz}$ frequency range, and we have been conducting research focusing on in vivo water by noninvasive measurements using dielectric spectroscopy. We have analyzed function expression mechanisms in vivo by non-invasive measurements with observation of the healing process of skin after burns [1], observation of changes in free water amount in the epidermis during exercise applied to sports medicine science [2], electric field analysis using dielectric models and various parts of the body for skin measurements [3-5], and so on. It has been also found that the electric field analysis of the open-end coaxial electrode can provide depth information from the skin surface.

Blood consists of cell components of RBCs and white blood cells, platelets, and plasma. It is reported that RBCs having particles of micrometer order size cause Maxwell-Wagner interface polarization, which can be observed by dielectric measurements in the $1 \mathrm{MHz}$ frequency region [6]. Blood flow measurements in vivo using open-ended electrodes have also shown that one of the observed relaxation processes is attributed to blood flow information [7].

A blood test can diagnose illness as a relatively light physical examination, though the test involves pain during blood collection. However, blood tests have a relatively low physical burden, even if it is relatively low as compared to other detailed examinations likes endoscopes. If the condition of blood can be observed by noninvasive and nondestructive dielectric measurement, the burden can be further reduced. More detailed discussion of the relaxation process observed in dielectric measurement is necessary for the application. In this study, in order to evaluate blood flow by dielectric spectroscopy measurements, blood flow measurements in vivo were performed using ultrasonic blood flow meter and laser blood flow meter in addition to dielectric measurements. It was discussed whether dielectric spectroscopy is also useful for blood flow measurements in living body compared with other methods.

\section{MATERIALS AND METHOD}

2.1 Examinees

Four examinees' normal data are shown in the Table 1. Each measurement was started after sprint stress in the room (temperature: $20.0 \pm 0.5^{\circ} \mathrm{C}$, humidity: $50.0 \pm 0.5 \%$ ).

Table 1. Characterizations of examinees and the normal data.

\begin{tabular}{|l|l|l|l|l|}
\hline & Gender & Age & $\begin{array}{l}\text { Blood } \\
\text { Pressure } \\
(\mathrm{mmHg})\end{array}$ & $\begin{array}{l}\text { Pulse } \\
(\mathrm{bpm})\end{array}$ \\
\hline A & Male & 25 & $116 / 74$ & 81 \\
\hline B & Female & 33 & $87 / 51$ & 62 \\
\hline C & Female & 23 & $88 / 60$ & 80 \\
\hline D & Male & 30 & $129 / 62$ & 76 \\
\hline
\end{tabular}




\subsection{Dielectric Measurements}

The dielectric measurements were performed in the frequency range from $40 \mathrm{~Hz}$ to $110 \mathrm{MHz}$ using a precision impedance analyzer (4294A, Agilent Technologies) and open-ended coaxial electrodes with the outer diameter $6.3 \mathrm{~mm}$ for skin above the wrist artery of the examinees $\mathrm{A}$ and $\mathrm{B}$.

\subsection{Laser Blood Flow Meter [8]}

The microcirculatory blood flow about $1 \mathrm{~mm}$ below the skin surface was measured for the examinee B using a laser blood flow meter (OMEGAWAVE, INC.) with $780 \mathrm{~nm}$ semiconductor laser. The measurement part was the fingertip of the left hand. Parameters obtained from the laser blood flow meter are values corresponding to the tissue blood volume, blood volume, and blood flow velocity. The laser blood flow meter processes the scattered light from the RBCs and detects the RBC number density and its velocity. The tissue blood flow rate is expressed as a value corresponding to the amount of blood flowing into a living tissue weight of $100 \mathrm{~g}$ per unit time. RBC number density corresponds to the blood volume, and the RBC flow velocity corresponds to the blood flow velocity.

\subsection{Ultrasonic Blood Flow Meter}

Mean blood flow velocity was obtained from the commercially available ultrasonic blood flow meter (ES-100V3, Hadeco) with $8 \mathrm{MHz}$ probe on skin above the wrist artery of the examinees B, C and D. This device measures the velocity of blood flow from the Doppler Effect.

\section{RESULTS AND DISCUSSION}

The dielectric relaxation curves obtained before and after sprint for two examinees are shown, respectively, in Figure 1, where the real part $\varepsilon$ ' and the imaginary part $\varepsilon$ " of the complex permittivity are plotted as a function of frequency $f$. It was confirmed that both examinees A and $\mathrm{B}$ returned to the dielectric relaxation curve before sprint with the change over time. It was found from the previous research that the spectrum shown in the $1 \mathrm{MHz}$ region reflects the interfacial polarization of the erythrocyte membrane [7].

Dielectric relaxation curved thus obtained can be described by the summation of four Cole-Cole equations [9] as

$$
\begin{aligned}
\varepsilon^{*} & =\varepsilon^{\prime}-j \varepsilon^{\prime \prime} \\
& =\varepsilon_{\infty}+\sum_{k=I}^{I V} \frac{\Delta \varepsilon_{k}}{1+\left(j \omega \tau_{k}\right)^{\beta}}-j \frac{\sigma_{D C}}{\varepsilon_{0} \omega}
\end{aligned}
$$

where $\varepsilon^{*}$ is the complex dielectric permittivity, $\varepsilon_{\infty}$ is the high frequency limit of the dielectric constant, $\Delta \varepsilon$ is the relaxation strength, $j$ is the imaginary unit, $\omega$ is the angular frequency, $\tau$ is the relaxation time, $\beta$ is the relaxation time distribution parameter, $\sigma_{D C}$ is the direct current (dc) conductivity, and $\varepsilon_{0}$ is the dielectric constant of vacuum, and I, II, III, and IV indicate respective processes in order from the higher frequency side. These processes are shown in Figure 2.
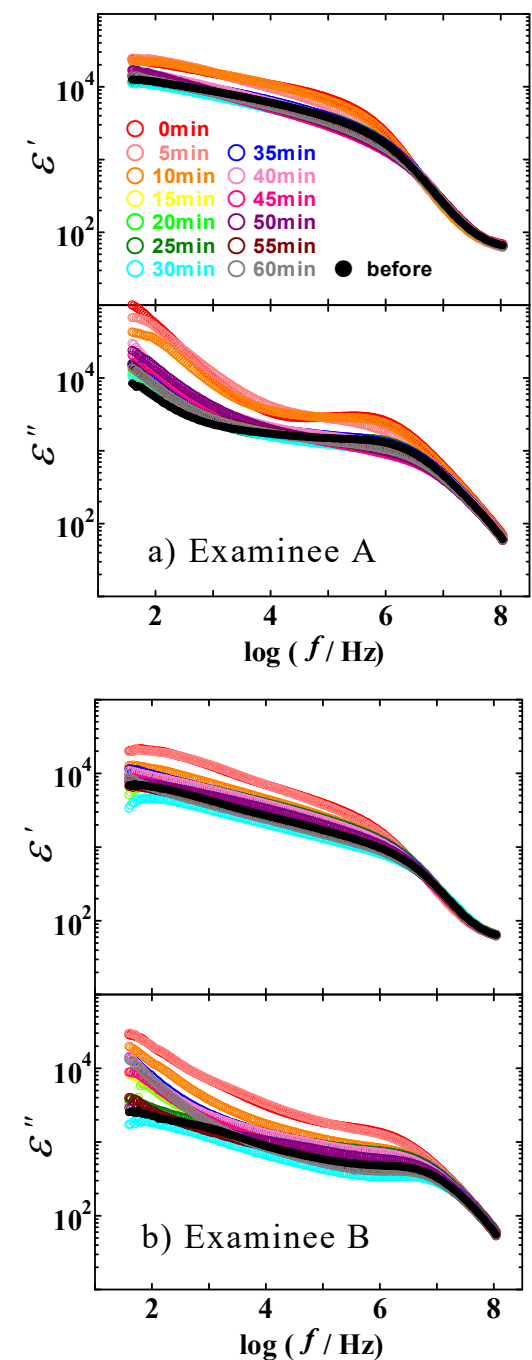

Figure 1. The time dependence of dispersion and loss curves obtained at just above artery of wrist. a) Examinee A, b) Examinee B.

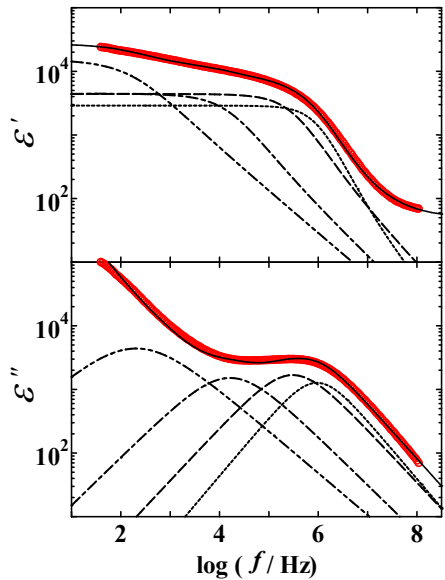

Figure 2. A typical example of the fitting procedure to data for examinee A before the sprint. Red solid line: Relaxation curve obtained by dielectric spectroscopy. Dot line: I-process. Dash line: II-process. Dot-dash line: III-process. Double-dot-dash line: IV-process. 


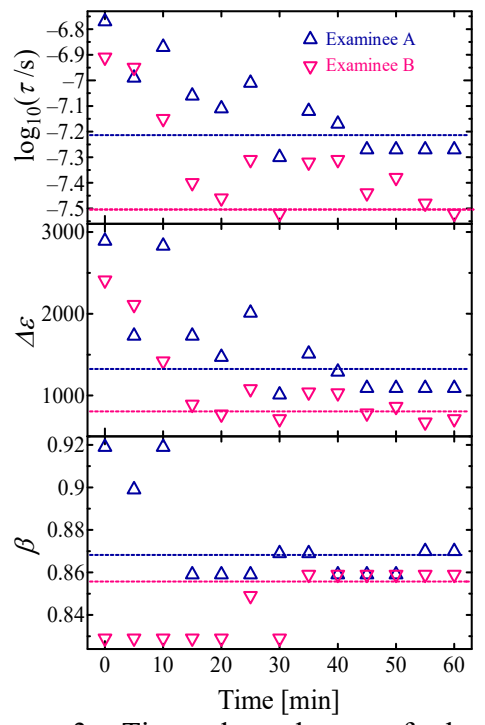

Figure 3. Time dependence of the relaxation parameters after the sprint. The dotted lines are the normal data for examinees.

Figure 3 shows the time dependences of relaxation parameters of relaxation process I after the sprint obtained from fitting procedures of Eq. 1 to the relaxation curve for each examinee. The dashed line shows the values of each examinee's relaxation parameters before the sprint. The relaxation strength $\Delta \varepsilon$ and the relaxation time $\tau$ are immediately increased after the sprint and returned to the resting value obtained before the sprint with the elapsed time for each examinee. Since relaxation process I shows the interfacial polarization of RBCs and fine blood vessels [7], an increase in the relaxation strength due to the sprint primarily indicates an increase in the RBC number. In addition, the relaxation time corresponds to the time scale of the polarization formation. So, the relaxation time of the interface polarization are corresponding to the length scale of the interface [10]. Therefore, it is considered that the interfacial polarization increased with the RBCs number due to the sprint are then decreased to a constant value with the elapsed time after the sprint. Although the relaxation time distribution parameter $\beta$ showed respective values for examinees, it was found that even if it changed due to the sprint, it approached the resting value with the elapsed time after the sprint.

Comparing the relaxation strengths obtained for two examinees, the examinee $\mathrm{B}$ has a smaller value than that for the examinee A. The lower relaxation intensity for examinee $\mathrm{B}$ compared to examinee $\mathrm{A}$ is considered to reflect the difference in the number of RBCs. Since the increase and decrease in interfacial polarization of RBC before and after exercise could be observed by dielectric spectroscopy the magnitude of relaxation intensity is greatly influenced by the number of RBCs. This is clear from the fact that the measurement of water content in the skin using the same electrode diameter at a site that is not affected by blood vessels has never produced such a difference of several hundred orders [11]. Furthermore, it is appropriate to consider that the time dependence of the relaxation processes cannot be affected by individual differences like subcutaneous fat and gender of

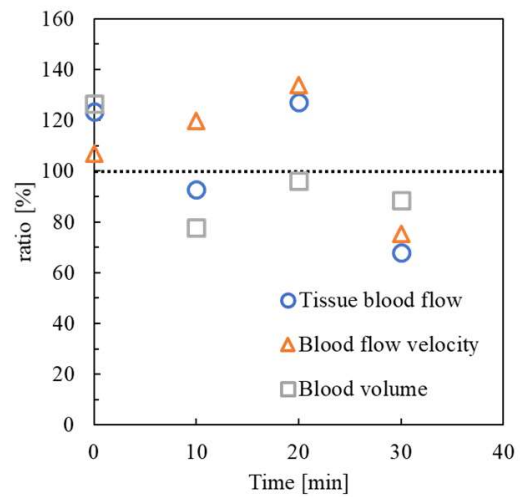

Figure 4. Time dependence of the blood flow parameter ratio for examinee $\mathrm{B}$.
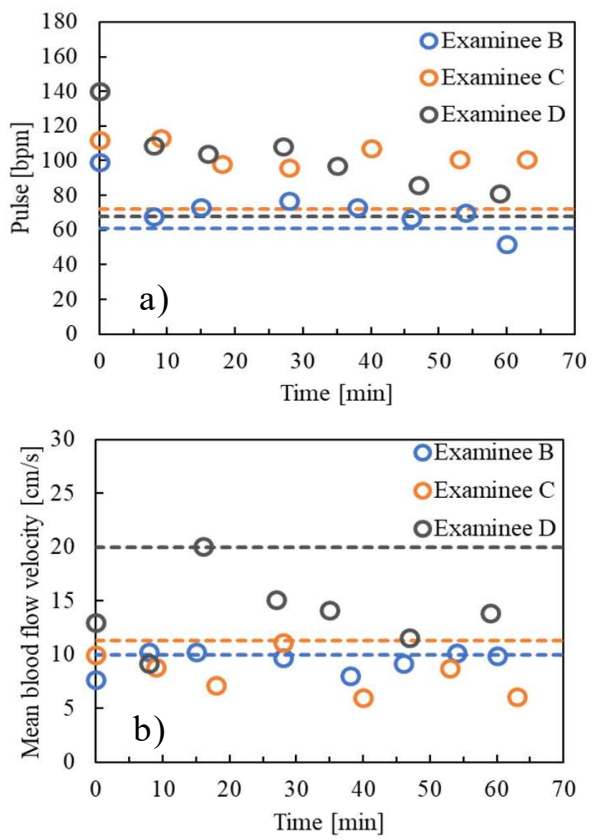

Figure 5. Time dependence of a) the mean blood flow velocity and $b$ ) the pulse. The dotted lines are the normal data for each examinee.

examinees. Therefore, the RBC count can be obtained also from the dielectric measurements.

Figure 4 shows the time dependence of the blood flow parameter ratio by laser blood flow meter for examinee B. The vertical axis represents the ratio to the value at rest and indicates the initial values were higher than $100 \%$ after the sprint. Since the blood volume corresponds to the RBC count density, it was confirmed that blood vessels dilated, and the blood volume was increased by the sprint.

Figure 5 shows the time dependence of the mean blood flow velocity and the pulse after the sprint for three examinees obtained by the ultrasonic blood flow meter. It was confirmed that the pulse of each examinee gradually returned to the values obtained before sprinting. The relationship between the pulse and the mean blood flow velocity is shown in Figure 6. It was found that the area plotted for each examinee was different. This result indicates that changes in the mean blood flow velocity due to the sprint reflect individual situations. 


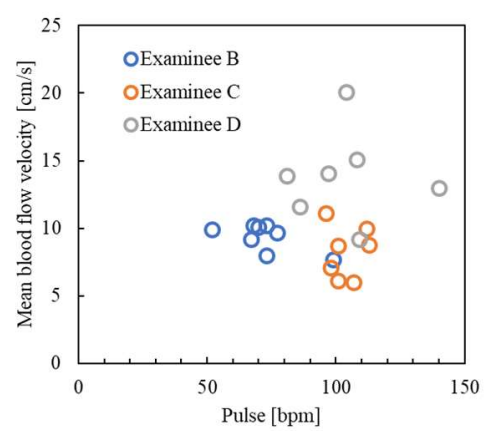

Figure 6. The relationship between the pulse rate and the mean blood flow velocity for each examinee.
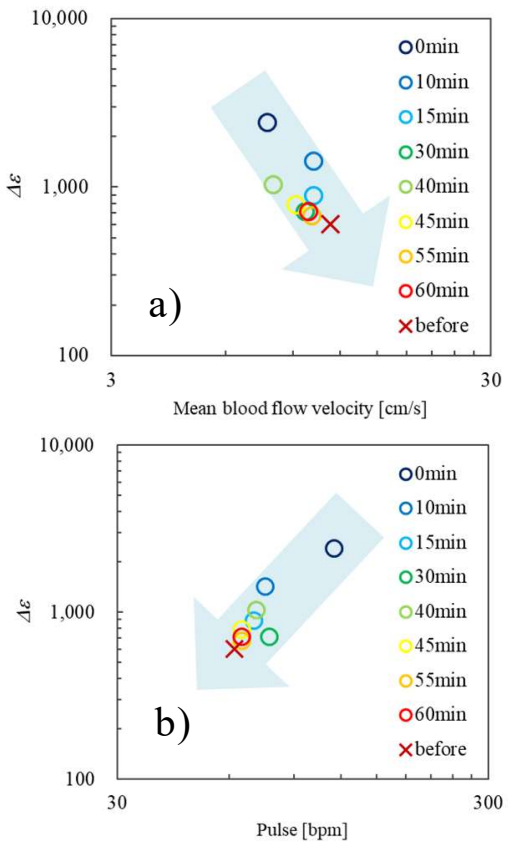

Figure 7. Comparison of the relaxation strength and ultrasonic blood flow, for examinee B.

a) the mean blood flow velocity and b) the pulse.

The arrow indicates the time dependence.

Figures $7 \mathrm{a}$ and $7 \mathrm{~b}$ show the comparison of the relaxation strength obtained from dielectric measurements from to mean blood flow velocity and pulse rate obtained with an ultrasonic blood flow meter, respectively, for examinee $\mathrm{B}$. Both the relaxation strength and pulse increased by the sprint and returned to resting values. The higher pulse delivered more blood, as confirmed by the increase in the relaxation strength corresponding to an increase in the number of RBCs. In addition, the mean blood flow velocity becomes slower after the sprint and returns to the resting velocity with the elapsed time, because the blood vessels are considered to be dilated by the sprint compared to the rest. It was found that the results of dielectric measurement and ultrasonic blood flow meter had well correlated each other.

This study clearly showed that blood conditions could be observed by non-invasive and non-destructive dielectric measurements with open-ended coaxial electrodes. Comparisons of the relaxation process I due to the interface polarization of the RBCs and fine blood vessels with results obtained from the ultrasonic blood flow meter and laser blood flow meter suggest a large possibility of the blood state evaluation in a living body by dielectric spectroscopy. In addition to the relaxation process I, other relaxation processes observed in dielectric measurements contain more information. By revealing these details, more blood information can be obtained than other approaches. It can be expected that dielectric spectroscopy will be able to obtain more detailed blood information from in vivo measurements in near future.

\section{CONCLUSION}

It was confirmed that the relaxation strength $\Delta \varepsilon$ and the relaxation time $\tau$ for a relaxation process observed around $1 \mathrm{MHz}$ in biometric dielectric spectroscopy for the wrist artery increasing during the sprint returned to the resting value obtained before the sprint with the elapsed time. These results indicate an increase in the number of RBCs observed during the sprint and were also confirmed by comparison with the results obtained by ultrasonic blood flow meter and laser blood flow meter. The amount of blood and the number of RBCs increases when a blood vessel dilates for the sprint. The blood flow rate was, however, found to be lower than that at rest because of the dilation of vessels. These results suggest that the blood state in the living body can be observed by dielectric spectroscopy, and even more details in the blood state should be brought from in vivo dielectric measurements.

\section{ACKNOWLEDGEMENT}

We are grateful to Associate Professor Haruchika Masuda, School of Medicine, Tokai University, for useful discussion. This work was approved as \#18050 "Research Plan for Human Subjects" established by Tokai University.

\section{REFERENCES}

[1] Y. Hayashi, N. Miura, N. Shinyashiki and S. yagihara, Phys. Med. Biol., 50, 599-612 (2005).

[2] T. Hashimoto, M. Yamamura, S. Yagihara, N. Shinyashiki, M. Kazami, I. Uchida, C. Sudou, K. Kouno, T. Arai, S. Osaki and S. Iwagaki, Tokai. J. Sports. Med. Sci. 15, 67-72 (2003).

[3] S. Naito M. Hoshi, S. Mashimo, Rev. Sci. Instrum., 67, 3633-3641 (1996).

[4] M. Hashimoto, T. Goto, N. Shinyashiki and S. Yagihara, Tokai. J. Sports. Med. Sci., 19, 52-62 (2007).

[5] T. Goto, M. Hashimoto, N. Shinyashiki, S. Yagihara and Y. Hayashi, Trans. Mat. Res. Soc. Japan., 31, 771-774 (2006).

[6] Y. Feldman, I. Ermolina, Y. Hayashi, IEEE Trans. Dielectr. Electr. Insul., 10. 728-753 (2003).

[7] T. Saito, H. Asano, H. Saito, R. Kita, N. Shinyashiki, and S. Yagihara, in preparation for publication.

[8] S. Kashima, Lasers. Life. Sci., 6, 79-90 (1994).

[9] K. S. Cole and R. H. Cole, J. Chem. Phys., 9, 341-351 (1941).

[10] K. Shoji, T. Saito, R. Kita, N. Shinyashiki, S. Yagihara, M. Fukuzaki, T. Ohzono, S. Nishimura, M. Hayashi and H. Tanaka, Trans. Mat. Res. Soc. Japan., 43, 201-204 (2018).

[11] Y. Maruyama, H. Kamata, S. Watanabe, R. Kita, N. Shinyashiki, S. Yagihara, Skin. Res. Technol., 26, 255-262 (2020). 